\title{
Characterizing digital image acquisition devices
}

\author{
Stephen E. Reichenbach, MEMBER SPIE \\ University of Nebraska-Lincoln \\ Computer Science and Engineering \\ Department \\ Lincoln, Nebraska 68588-0115 \\ Stephen K. Park \\ College of William and Mary \\ Computer Science Department \\ Williamsburg, Virginia 23185 \\ Ramkumar Narayanswamy \\ Science and Technology Corporation \\ Hampton, Virginia 23666
}

\begin{abstract}
Despite the popularity of digital imaging devices (e.g., CCD array cameras) the problem of accurately characterizing the spatial frequency response of such systems has been largely neglected in the literature. This paper describes a simple method for accurately estimating the optical transfer function of digital image acquisition devices. The method is based on the traditional knife-edge technique but explicitly deals with fundamental sampled system considerations: insufficient and anisotropic sampling. Results for both simulated and real imaging systems demonstrate the accuracy of the method.
\end{abstract}

Subject terms: image formation; image restoration; modulation transfer function; optical transfer function; point spread function; evaluation technology; image acquisition devices; sampling.

Optical Engineering 3012), 170-177 (February 1991).

\section{CONTENTS}

1. Introduction

2. Problems with the traditional knife-edge technique 2.1. Noise

2.2. Undersampling

2.3. Anisotropic sampling

3. Knife-edge method for digital devices

3.1. One-dimensional estimates

3.2. Two-dimensional estimates

4. Simulations

5. Experimental results

6. Conclusion

7. Acknowledgments

8. References

\section{INTRODUCTION}

Despite the growing popularity of digital imaging devices, the problem of accurately estimating the spatial frequency response or optical transfer function (OTF) of these devices has been largely neglected. Traditional methods for estimating OTFs were designed for film cameras and other devices that form continuous images. These traditional techniques do not provide accurate OTF estimates for typical digital image acquisition devices because they do not account for the fixed sampling grids of digital devices. This paper describes a simple method for accurately estimating the OTF of a digital image acquisition device. The method extends the traditional knife-edge technique ${ }^{1-13}$ to account for sampling.

One of the principal motivations for digital imaging systems is the utility of digital image processing algorithms, many of which require an estimate of the OTF. Algorithms for enhancement, spatial registration, geometric transformations, and other purposes involve restoration-removing the effects of the image acquisition device. Nearly all restoration algorithms (e.g., the

Invited paper EV-102 received Sept. 20, 1989; revised manuscript received May 4, 1990; accepted for publication Aug. 13, 1990.

(C) 1991 Society of Photo-Optical Instrumentation Engineers inverse filter, the Wiener filter, and the constrained-least-squares filter) are conditioned on a linear, shift-invariant model of the acquisition device characterized by the OTF. The performance of these algorithms can be degraded, sometimes dramatically, by inaccurate OTF estimates.

The knife-edge technique computes the OTF estimate from an image of a knife edge (a target with a straight-edged, sharp discontinuity, i.e., a step function). A knife-edge target is relatively easy to fabricate accurately - much easier than the targets required by other techniques (e.g., an infinitestimal pulse, a set of sinesoidal targets, or a set of bar targets). Only one knife edge is needed and, with care, radiance nonuniformity and edge irregularity can be made small. Also, in some situations (e.g., remote sensing), the OTF must be estimated without the use of specially fabricated targets. Because sharp edges occur naturally in many scenes, the knife-edge technique frequently can be used even if an image of a special target is not available.

As is demonstrated in this paper, if the traditional knife-edge technique is used to characterize a typical imaging device, sampling effects cause significant errors in the OTF estimate. The knife-edge technique was originally developed for characterizing acquisition devices that produce spatially continuous (rather than digital) images ${ }^{1}$ (especially film cameras ${ }^{2,6,7,9,10}$ ). The traditional knife-edge technique relies on oversampling a continuous image with a scanning device. If the image is not oversampled, as in a typical digital image, then the traditional knife-edge estimate is inaccurate.

The technique developed in this paper solves the problems caused by sampling and accurately estimates the OTFs of digital image acquisition devices, even those that inherently undersample. Section 2 describes the traditional knife-edge technique and details the problems of applying it to digital imaging devices. Section 3 describes our extension of the traditional knife-edge technique to deal with sampling. In Section 4, the extended knife-edge technique is applied to images generated by a simulated system. In Section 5, the extended knife-edge technique is used to characterize a CCD digital camera. 

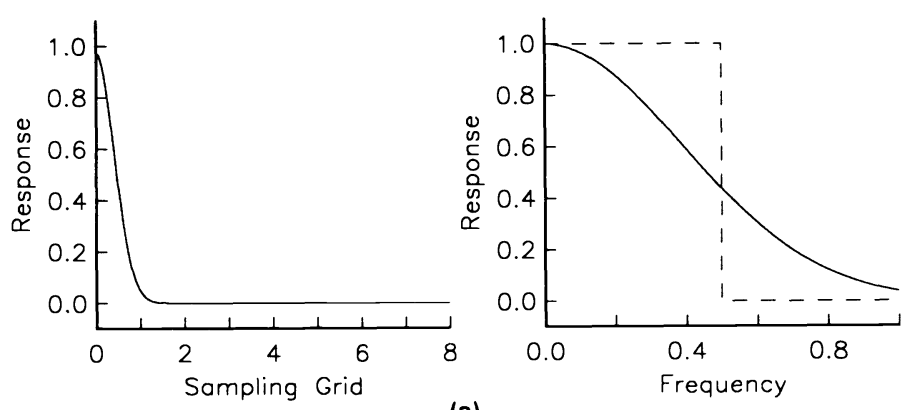

(a)
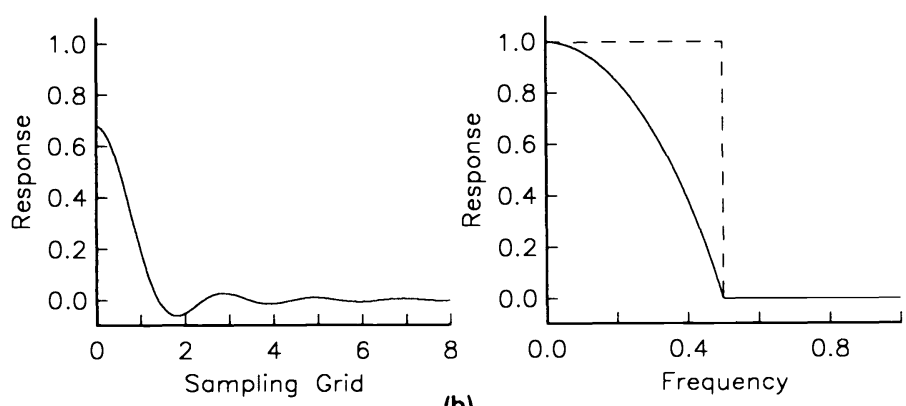

(b)
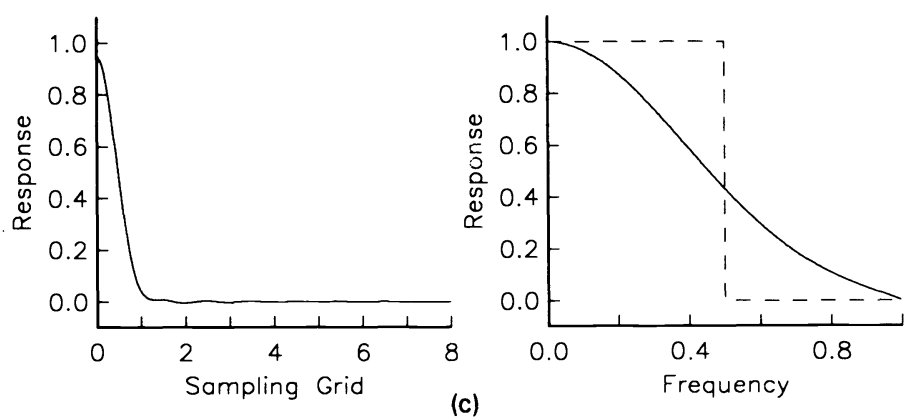

Fig. 1. Example system and estimates. (a) Example system PSF and OTF ( $\rho_{c}=0.55$ ). (b) Traditional knife-edge estimate. (c) Improved knife-edge estimate.

\section{PROBLEMS WITH THE TRADITIONAL KNIFE- EDGE TECHNIQUE}

A traditional "analog" image acquisition device produces an image that is spatially continuous. To characterize the spatial frequency response of such a device, the continuous image of a knife edge is scanned and sampled (e.g., with a microdensitometer ${ }^{6}$ ) along a line that is perpendicular to the edge. The sampled data are a measure of the device's edge spread function (ESF). The derivative of the ESF with respect to the scan direction is the line spread function (LSF). The Fourier transform of the LSF is a one-dimensional slice through the center of the two-dimensional OTF. ${ }^{1}$ When the knife-edge technique is applied to a continuous image, the principal problem is noise.

Although noise is also a problem in estimating the OTF of a digital device, there are other problems as well. In a digital image acquisition device, image formation (i.e., the operation of the OTF on the scene) is followed by sampling at fixed points of the image. The fixed sampling grid of a digital device presents two problems for the knife-edge technique: insufficient and anisotropic sampling. In the traditional knife-edge technique, the scans of the continuous images are sampled to facilitate processing (i.e., computing the derivative). However, because sam- pling is implemented with an external device, the sampling rate and orientation relative to the image can be controlled. In contrast, the sampling grid of a digital device is fixed and neither its density nor orientation relative to the image can be changed.

\subsection{Noise}

Noise corrupts the ESF in actual edge scans. Differentiation of the noisy scans to estimate the LSF amplifies the high-frequency components of the scans, where the signal-to-noise ratio (SNR) is typically lowest. Many techniques have been proposed to deal with noise (e.g., Refs. 6, 9, 11, and 12). Some of these techniques implicitly affect the OTF estimate by suppressing the estimate where noise is high or presuming a parametric form for the OTF.

Implemented properly, scan-line averaging is a simple technique that suppresses zero-mean noise without affecting the OTF estimate. Scan-line averaging is implemented by generating many scans across an edge. Then, the scans are registered using estimates of the edge location (i.e., the scans are shifted so that the edge points in all of the scans are aligned) and averaged. ${ }^{11}$ The extended knife-edge technique described in Sec. 3 uses scan-line averaging.

\subsection{Undersampling}

Typical digital image acquisition devices are designed to undersample. ${ }^{94}$ (In practice, sufficient sampling can be achieved only with a design that causes excessive blurring.) Undersampling causes aliasing - the folding of spatial-frequency components above the Nyquist frequency into frequencies below the Nyquist frequency.

When the traditional knife-edge technique is applied to typical digital imaging devices, aliasing causes significant errors in the OTF estimate. For example, consider a simulated, one-dimensional system with a Gaussian (presampling) OTF:

$\hat{h}(u)=\exp \left(-\frac{u^{2}}{\rho_{c}^{2}}\right)$,

where $u$ is the spatial frequency (normalized to the sampling rate) and $\rho_{c}=0.55$. The OTF and the corresponding point spread function (PSF) are pictured in Fig. 1(a). (The PSF is the inverse Fourier transform of the OTF.) Note that the response of the OTF extends to frequencies beyond the sampling passband or Nyquist frequency $(u=0.5)$. This is typical-most digital imaging systems have a significant response to frequencies beyond the sampling passband (although sampling causes these frequencies to alias)

Figure 1(b) pictures the traditional knife-edge estimate of the OTF in Fig. 1(a) derived from a noise-free edge scan. The OTF estimate is cut off at the Nyquist frequency and is inaccurate at all but the lowest frequencies. The sharp cutoff in the OTF estimate appears as ringing in the corresponding PSF, and the loss of high frequencies in the OTF estimate is seen as a broadening or loss of sharpness in the PSF.

As described in Sec. 3, the extended knife-edge technique uses many edge scans to generate a single scan with superresolution (resolution higher than the sampling rate) sufficient to eliminate aliasing. For the example system of Fig. 1(a), doubling the sampling rate sufficiently samples the ESF. The knifeedge estimates of the OTF and PSF based on twofold superresolution are shown in Fig. 1(c). It is evident that in this case, 
the extended knife-edge technique produces a much more accurate estimate than the traditional knife-edge technique.

Sample/scene phase $e^{15,16}$ (the location of the edge relative to the sample points) is a source of variability in the edge scans. In sufficiently sampled scans, differences between scans caused by sample/scene phase shifts can be removed by spatial interpolation. ${ }^{11}$ However, interpolation cannot remove variations caused by sample/scene phase shifts from undersampled scans. If the traditional knife-edge technique is applied to insufficiently sampled edge scans, the OTF estimate varies with sample/scene phase. The extended knife-edge technique described in Sec. 3 achieves sufficient sampling, so sample/scene phase shifts can be removed.

\subsection{Anisotropic sampling}

The knife-edge technique is fundamentally one-dimensionalthe estimate is a one-dimensional slice through the center of the two-dimensional OTF. Many traditional optical systems have a radially symmetric OTF that can be characterized by a onedimensional slice. If needed, other slices of the OTF can be obtained by rotating the knife-edge target and acquiring additional images. For analog imaging systems, as the knife edge is rotated, the scanning device and the continuous image can be rotated relative to one another so that the scan direction remains perpendicular to the knife edge in the image regardless of the edge orientation.

In a digital imaging system, the sampling grid is fixed and cannot be rotated relative to the image. This is a problem because the sampling grid is rotationally anisotropic - the density of the sampling points along lines perpendicular to the knife edge varies with the angle of rotation. Moreover, two-dimensional estimates are more important for digital imaging systems because the (nonoptical) electronic components often cause radial asymmetry in the OTF. ${ }^{13,16,17}$ As described in Sec. 3, despite sampling anisotropy, the extended knife-edge technique can be applied to an edge at nearly any angle.

\section{KNIFE-EDGE METHOD FOR DIGITAL DEVICES}

In this section, the traditional knife-edge technique is extended to deal with undersampling and anisotropic sampling in digital systems. By averaging many registered scans, the extended method generates a superresolution scan with sufficient sampling density and high SNR from which the OTF can be accurately estimated. The one-dimensional method is described in Sec. 3.1. As described in Sec. 3.2, this method can be applied at nearly any angle to generate two-dimensional estimates.

\subsection{One-dimensional estimates}

To achieve superresolution, the knife edge is aligned slightly off-perpendicular to the scan direction, as in Fig. 2(a). Tescher and Andrews ${ }^{11}$ characterized this unavoidable off-perpendicular alignment as unfortunate, but that is not the case for digital devices-it is the key to increasing the effective sampling rate. Figure 2(b) shows the noise-free edge responses and sample points for this sequence of scans. The location of the edge shifts slightly from scan to scan.

Modeled mathematically, the value of pixel $m$ in scan $n$ of image $p$ is

$p_{n}(m)=\int_{-\infty}^{\infty} s\left(m-x^{\prime}-x_{n}\right) h\left(x^{\prime}\right) d x^{\prime}+e_{n}(m)$,

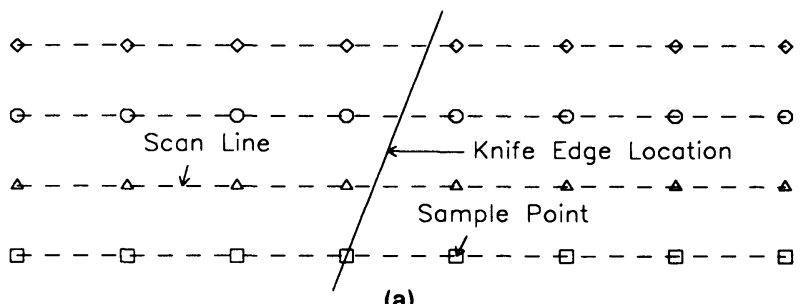

(a)

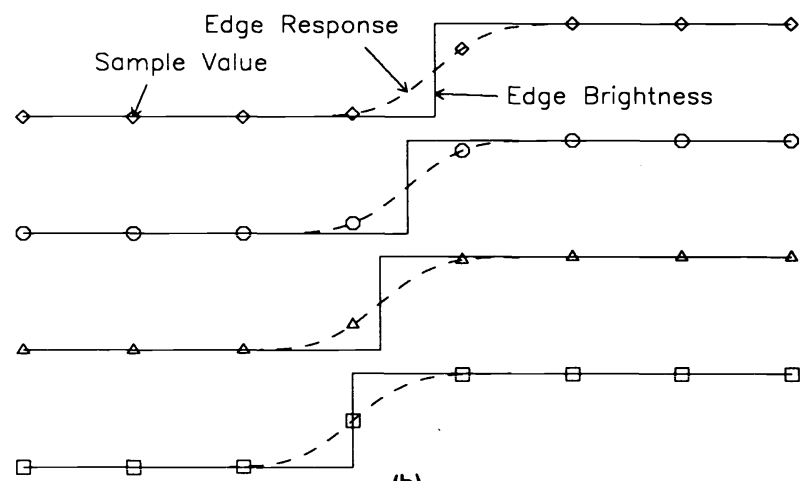

(b)

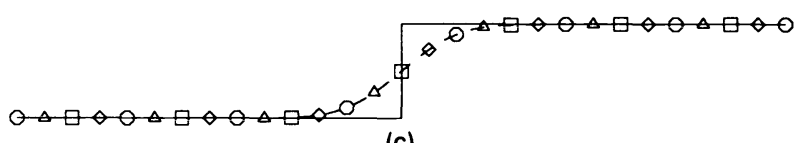

(c)

Fig. 2. Registering scans with shifted knife edge. (a) Sampling grid with knife edge skewed from perpendicular. (b) Knife-edge shift in successive scans. (c) Combined scan with registered edges.

where $s$ is the knife-edge target with the step edge located at $x_{n}$ in scan $n, h$ is the (unknown) PSF, and $e_{n}$ is the noise. The coordinates are normalized to the sampling interval.

If the individual scans are correctly registered according to the location of the edge, then as illustrated in Fig. 2(c), the combined scan generated by the superposition of the registered scans will contain more edge response samples than any single scan, thereby effectively increasing the sampling resolution.

Correctly registering scans requires estimating the edge location in each scan line to subpixel accuracy. Several methods have been suggested for estimating step-edge locations to subpixel accuracy. ${ }^{18-21}$ Any of these methods can be used. We estimate the knife-edge location as follows: (1) estimate the edge location in each scan line, (2) use linear regression to fit a line through the individual edge estimates, and (3) use the regression line to improve the estimate of the edge location in each scan line. Steps (2) and (3) rely on the straightness of the edge and should not be employed if the knife edge is irregular.

For noise-free scans and perfect registration [as in Fig. 2(c)], in the continuum limit as the number of scan lines increases, the combined scan $q$ is

$q(x)=\int_{-\infty}^{\infty} s\left(x-x^{\prime}\right) h\left(x^{\prime}\right) d x^{\prime}$,

and characterizing the system to any resolution involves a straightforward inversion of this equation to solve for the PSF $h$. In real scans, noise is a problem, registration is imperfect, and resolution is necessarily limited by the finite number of scans used. These problems are illustrated in the combined scan $q$ 


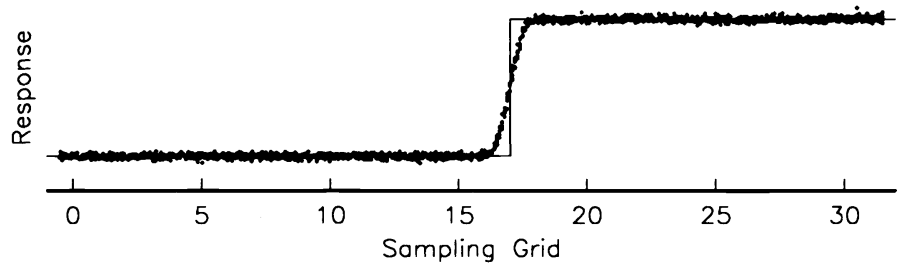

(a)

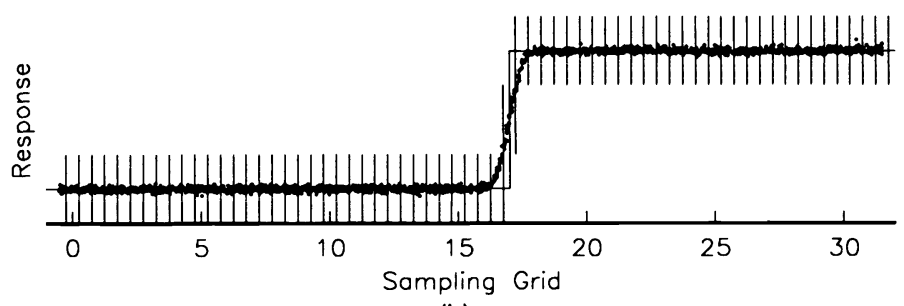

(b)

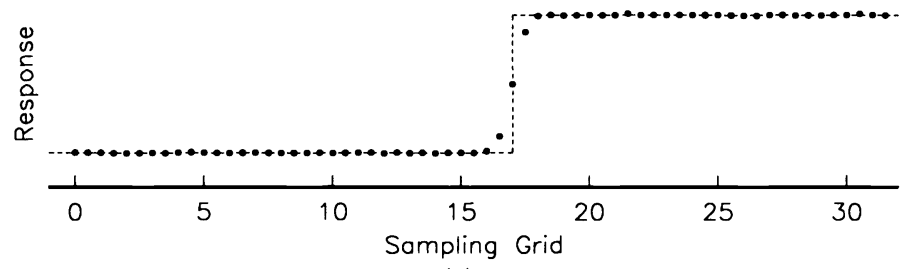

(c)

Fig. 3. Averaging and resampling. (a) Registered noisy scans. (b) Averaging intervals along the combined scan. (c) Resampled averaged scan.

pictured in Fig. 3(a). This combined scan consists of 64 noisy scans registered by estimated edge location.

The noise in the combined scan can be suppressed by smoothing and resampling, thereby sacrificing some spatial resolution in order to reduce noise. The resampling resolution should be at least as great as the OTF cutoff, and the smoothing filter should be matched to the resampling resolution. For typical digital image acquisition devices, twofold (or slightly higher) superresolution is usually sufficient.

Averaging is a simple smoothing operation that is easily computed for the irregularly spaced samples of the combined scan. If the resampling resolution is $\alpha$ and the width of the averaging interval is $1 / \alpha$, then each of the original samples contributes to exactly one resampled value. For example, Figs. 3(b) and 3(c) illustrate an averaging interval of $1 / 2$ and a resampling resolution (or superresolution ratio) of $\alpha=2$.

Assuming the sample/scene phase is uniformly distributed over all scans, in the continuum limit, the averaged and resampled scan $r$ is

$r\left(\frac{m}{\alpha}\right)=\alpha \int_{-\infty}^{\infty} \Pi(m-\alpha x) q(x) d x$,

for all integers $m$, where $\square$ is the unit pulse. (Note that the sample points are indexed by the integer $m$, but are located at intervals of $1 / \alpha$.) If the noise is zero mean and the scan lines are registered into the correct averaging interval, then from Eqs. (3) and (4), in the continuum limit as the number of scans increases, the resampled scan is

$r\left(\frac{m}{\alpha}\right)=\alpha \int_{-\infty}^{\infty} \Pi(m-\alpha x)\left[\int_{-\infty}^{\infty} s\left(x-x^{\prime}\right) h\left(x^{\prime}\right) d x^{\prime}\right] d x$.

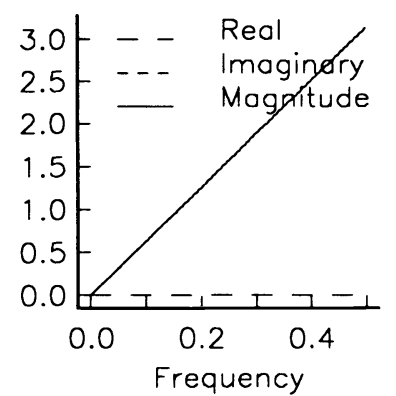

(a)

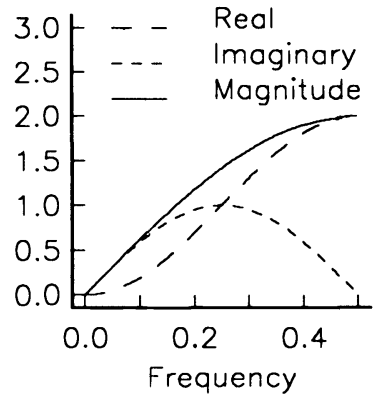

(b)

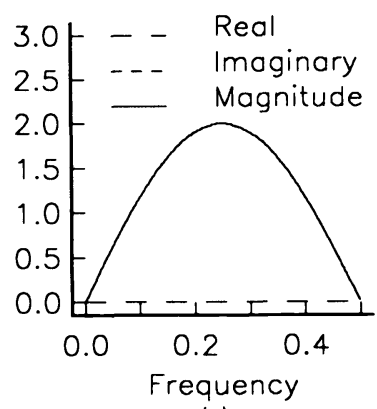

(c)

Fig. 4. Spatial derivatives in the Fourier frequency domain. (a) Exact derivative. (b) $\left[\begin{array}{ll}-1 & 1\end{array}\right]$ approximation. (c) $\left[\begin{array}{lll}-1 & 0 & 1\end{array}\right]$ approximation.

Under these conditions, when a large number of scans are used, sample/scene phase bias, residual noise, and misregistration errors are negligible.

Recovering the OTF from Eq. (5) is an inverse problem. The inverse function of the step edge $s$ is the derivative operator with respect to the normal to the edge (the direction of the scan). As is shown in Fig. 4, commonly used discrete approximations of the derivative (e.g., $\left[\begin{array}{ll}-1 & 1\end{array}\right]$ and $\left.\left[\begin{array}{lll}-1 & 0 & 1\end{array}\right]\right)$ suppress high frequencies. Therefore, using a discrete-difference approximation of the derivative to invert Eq. (5) attenuates high frequencies of the OTF estimate. Accurate inversion is simpler in the frequency domain. The frequency domain equivalent of $\mathrm{Eq}$. (5) is

$\hat{r}(u)=\operatorname{sinc}\left(\frac{u}{\alpha}\right) \hat{s}(u) \hat{h}(u), \quad|u|<\frac{\alpha}{2}$,

where the sinc function is the spectrum (or Fourier transform) of the unit pulse,

$\operatorname{sinc}(u)= \begin{cases}1, & \text { if } u=0, \\ \frac{\sin (\pi u)}{\pi u}, & \text { otherwise },\end{cases}$

$\hat{s}$ is the spectrum of the knife-edge target $s$,

$\hat{s}(u)= \begin{cases}\frac{1}{2}, & \text { if } u=0, \\ \frac{-i}{2 \pi u}, & \text { otherwise },\end{cases}$

and $\hat{h}$ is the OTF being estimated. Then, the OTF estimate is

$\hat{h}(u)=\frac{\hat{r}(u)}{\operatorname{sinc}(u / \alpha) \hat{s}(u)}$. 


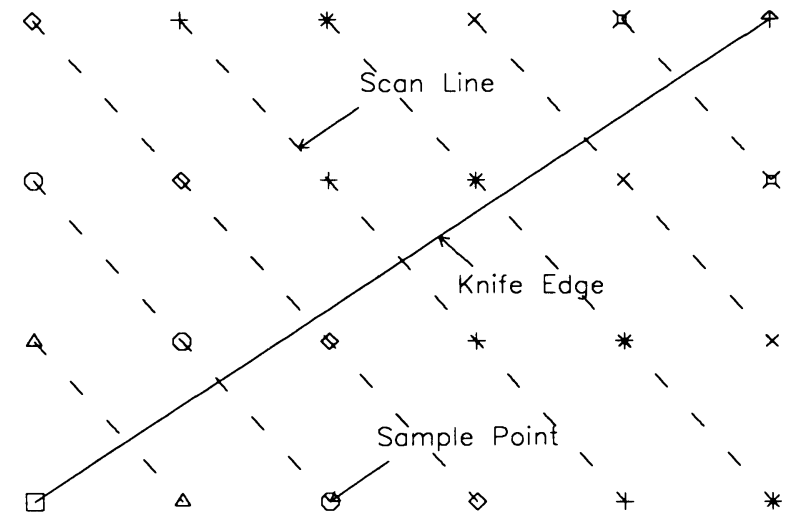

Fig. 5. Diagonal knife-edge scans.

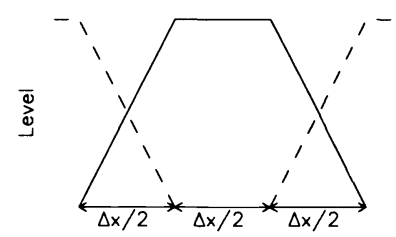

Horizontal Response

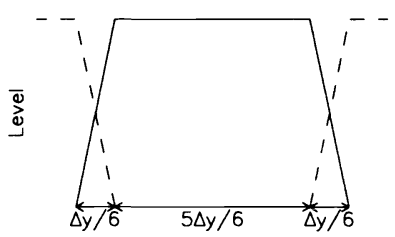

Vertical Response
Fig. 6. Idealized horizontal and vertical spatial responses.

In practice, $\hat{r}$ is computed as the DFT of the smoothed, resampled scan $r$ and the OTF estimate in Eq. (9) is computed only at discrete frequencies of the DFT. The use of the DFT assumes the periodic extension of a finite-length, discrete scan. Generally, the impact of using a finite-length scan is negligible because the PSF is much narrower than the scan length. ${ }^{8}$ Periodicity can be effected by using a wide bar with a knife edge on both sides or (if the OTF is symmetric) mirroring the edge scan.

To summarize, the extended knife-edge technique is as follows:

(1) Generate many scan lines across a knife edge that is oriented nearly perpendicular to the scan direction. (A slant of at least one pixel across all scans is required to produce a uniform distribution of sample/scene phase.)

(2) Register the scans according to their estimated edge locations.

(3) Choose a superresolution (or resampling) ratio $\alpha$.

(4) Average the registered scan values that fall within subpixel intervals of width $1 / \alpha$ about the resampling points.

(5) Compute $\hat{r}$, the DFT of the resampled scan.

(6) Compute $\hat{h}$, the estimate of the OTF slice [as in Eq. (9)], to the superresolution Nyquist frequency $( \pm \alpha / 2)$.

\subsection{Two-dimensional estimates}

A two-dimensional OTF estimate can be reconstructed from many one-dimensional slices at different angles. Despite sampling anisotropy, the extended knife-edge technique can be applied at nearly any angle. Figure 5 illustrates how to generate scans at $45^{\circ}$ across an edge. Each scan consists of samples from different rows and columns. Scans at other off-axis angles can be implemented similarly. Note that the sampling rate along the scan is a function of the angle. For example, at $45^{\circ}$, as in Fig. 5 , the sampling rate is $1 /\left[(\Delta x)^{2}+(\Delta y)^{2}\right]^{1 / 2}$, where $\Delta x$ and $\Delta y$ are the horizontal and vertical sampling rates. The differences in sampling rates must be considered in reconstructing a twodimensional OTF.

Before proceeding with the involved process of two-dimensional reconstruction, it is sensible to analyze the system for radial symmetry and separability. If the horizontal, vertical, and diagonal OTF slices are nearly identical, it is indicative (but not conclusive) evidence that the system is symmetric. Likewise, if the product of the horizontal and vertical slices is nearly the same as the diagonal, it suggests (but does not prove) that the system is separable. These assessments require only three slices, and if either conclusion is accepted, the process of acquiring other scans and reconstructing can be avoided.

\section{SIMULATIONS}

This section presents simulation results for the extended knifeedge technique. The digital acquisition device is modeled with two components: the camera optics and the sensor array. The camera optics are modeled with a two-dimensional Gaussian OTF:

$\hat{h}_{o}(u, v)=\exp \left[-\left(\frac{\sqrt{u^{2}+v^{2}}}{\rho_{c}}\right)^{2}\right]$,

where $\rho_{c}=1 / \sqrt{2}$. This OTF component is both symmetric and separable. The sensor array model assumes rectangular potential wells with unit brightness response within wells and linear falloff between wells. ${ }^{22,23}$ The sensor PSF is pictured in Fig. 6. The sensor array OTF is

$\hat{h}_{d}(u, v)=\operatorname{sinc}\left(\beta_{x} u\right) \operatorname{sinc}\left(\gamma_{x} u\right) \operatorname{sinc}\left(\beta_{y} v\right) \operatorname{sinc}\left(\gamma_{y} v\right)$,

where $\beta_{x}$ and $\beta_{y}$ are the dimensions of the potential wells and $\gamma_{x}$ and $\gamma_{y}$ are the distances between wells. For the simulations in this section, $\beta_{x}=5 / 6, \gamma_{x}=1 / 6, \beta_{y}=1 / 2$, and $\gamma_{y}=1 / 2$. The sensor OTF component is separable but not symmetric. The composite OTF model is the product of the OTFs of the optics and the second array:

$\hat{h}(u, v)=\hat{h}_{o}(u, v) \hat{h}_{d}(u, v)$.

The knife-edge targets were simulated with three superresolution digital images (digital images with greater resolution than the sampling grid). Three $1024 \times 1024$ digital scenes were used: one with a vertical bar, one with a horizontal bar, and one with a diagonal bar. In each scene, the bar was slightly sloped relative to the scan direction (a slope of 1 scene element per 64 scan lines). Each of these targets provided two knife edges (one on each side of the bar). The effect of the system on the scene is simulated by multiplying the DFT of the digital scene by the OTF. The inverse DFT of this product is the simulated image. To simulate sampling, the images of the horizontal and vertical bars were resampled in the scan direction at every sixteenth scenel (scene element) to produce 1024, 64-element edge scans. The image of the diagonal bar was sampled both horizontally and vertically to produce 128 edge scans of varying length. In all three images, border effects corrupt the top and bottom scans, so only half the edge scans were retained (those in the center). White noise was then added to these edge scans.

The image SNR is the ratio of the step-edge height to the contrast (standard deviation) of the noise: 


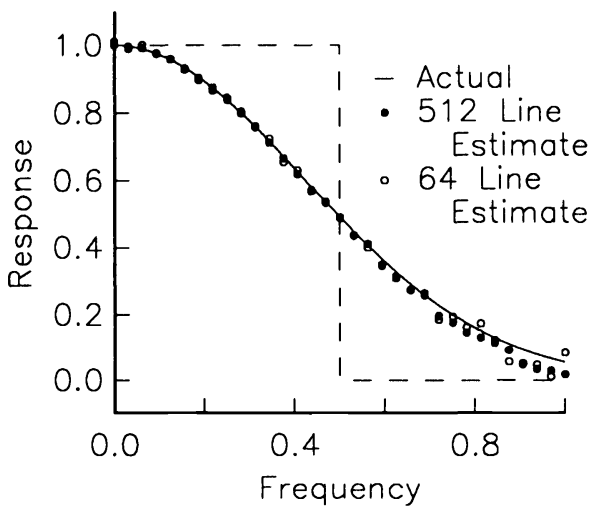

(a)

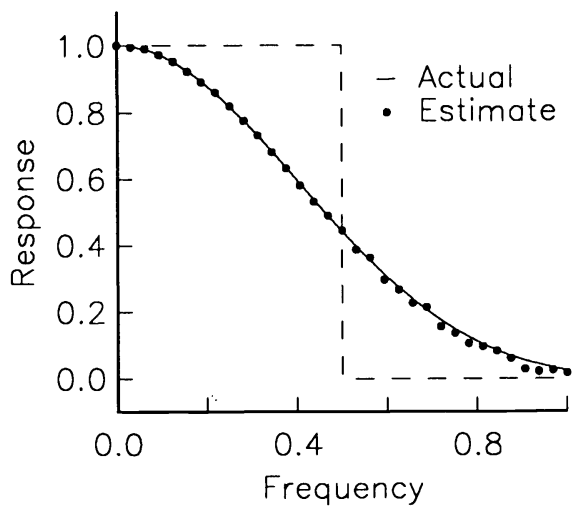

(b)

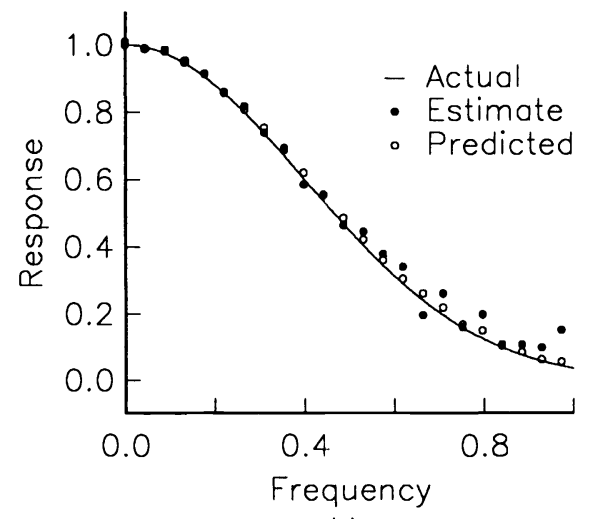

(c)

Fig. 7. Simulation results. (a) Horizontal OTF and estimates. (b) Vertical OTF and estimate. (c) Diagonal OTF, estimate, and predicted.

image SNR $\triangleq \frac{|o-b|}{\sigma_{e}}$,

where $o$ is the brightness value of the object (i.e., the bar) and $b$ is the brightness value of the background. The simulation was run for SNR values of $16,32,64,128$, and 256 as well as no noise. For example, the registered scans in Fig. 3(a) have SNR $=64$. For each noise level, the OTF was estimated from 2, 4, $8,16,32,64,128,256$, and 512 scan lines. In all of the experiments, the OTF estimates were made with $\alpha=2$. For each experiment, the mean-square error (MSE) of the OTF estimate relative to the energy of the actual OTF is

relative MSE $\triangleq \frac{\sum_{\mu}\left|\hat{h}(\mu)-\hat{h}_{\mathrm{est}}(\mu)\right|^{2}}{\sum_{\mu}|\hat{h}(\mu)|^{2}}$.

In the simulation, the MSE of the OTF estimate is conditioned on the image SNR and the number of scan lines used. The experimental results bear out the expected relationships. For a fixed number of scan lines, the MSE varies inversely with the square of SNR (e.g., doubling SNR cuts MSE by a factor of 4). For fixed SNR, the MSE varies as the inverse of the number of lines (e.g., doubling the number of lines halves the MSE). In practice, the SNR is not easily controlled, so the MSE of the estimate depends principally on the number of lines used.

Figure 7(a) shows the horizontal OTF (the solid line) and the OTF estimates for SNR = 64 based on both 64 scan lines (open circles) and 512 scan lines (closed circles). For the estimate based on 64 scan lines, the relative MSE (within the band of frequencies estimated) is approximately $6 \times 10^{-4}$. For 512 scan lines the MSE is about $8 \times 10^{-5}$. Figure 7(b) shows the vertical OTF and the estimate based on 512 scan lines. Figure 7(c) shows the diagonal OTF, the estimate based on 512 scan lines (closed circles), and the response predicted by the product of the horizontal and vertical estimates (open circles).

None of the estimates is very different from the others, indicating that the two-dimensional OTF is nearly symmetric. The product of the vertical and horizontal estimates is similar to the diagonal knife-edge estimate, supporting the hypothesis that the system is separable. It is clear from these estimates that $\hat{h}_{o}$, the Gaussian component of the simulated OTF associated with the optics, dominates $\hat{h}_{d}$, the OTF component associated with the detector.

\section{EXPERIMENTAL RESULTS}

This section presents results of the extended knife-edge technique applied to a real digital camera. A traditional knife-edge estimate of the OTF of this system would be inaccurate below the Nyquist frequency and would not measure the components above it. The superresolution estimate derived by the extended knife-edge technique is consistent with conventional OTF design of digital imaging systems and with measured responses to a square-wave target.

The extended knife-edge procedure was used to estimate the OTF of a Photometrics $183 \mathrm{~S}$ slow-scan, three-stage, thermoelectric-cooled CCD camera system with a Texas Instruments 4849 array sensor and a Pentax $1.850 \mathrm{~mm}$ lens. A commercial test target with a black square printed on white Mylar was placed as close to the camera as could be focused $(45 \mathrm{~cm})$. The $f$-stop was set to 9.5 and the exposure time was $0.1 \mathrm{~s}$. The resulting images were corrected for dark-field and flat-field response as

$p^{\prime}(m, n)=\frac{p(m, n)-d(m, n)}{f(m, n) / \bar{f}}$,

where $d(m, n)$ is the dark-field response (the CCD output with the shutter closed), $f(m, n)$ is the flat-field response (the response to a target of uniform reflectance), and $\bar{f}$ is the mean of the flat-field image. Images with 372, 64-pixel scans across the vertical and horizonal bars were cut from the full $388 \times 584$ images. The image of the diagonal bar was cut to $128 \times 128$.

Figure 8 illustrates the OTF estimate (for $\alpha=2$ ). The response is typical for digital imaging systems designed to balance the tradeoff between blurring and aliasing - some attenuation of frequencies below the Nyquist frequency that will cause blurring and some response above the Nyquist frequency that will result in aliasing. The magnitude of the OTF at the Nyquist limit is slightly greater than 0.3 . Noise begins to dominate the OTF estimate near the sampling frequency, where the OTF magnitude and the SNR are small.

The vertical, horizontal, and diagonal responses are not very much different from one another. This is evidence that the OTF is nearly circularly symmetric. The product of the vertical and horizontal transfer function estimates is a poor estimate of the diagonal response at the appropriate frequency. This indicates that the system function is not separable.

The OTF estimates for this camera were corroborated using an image of a bar chart that conforms to NBS 1010A and ANSI/ ISO \#2 standards taken under the conditions described. The 


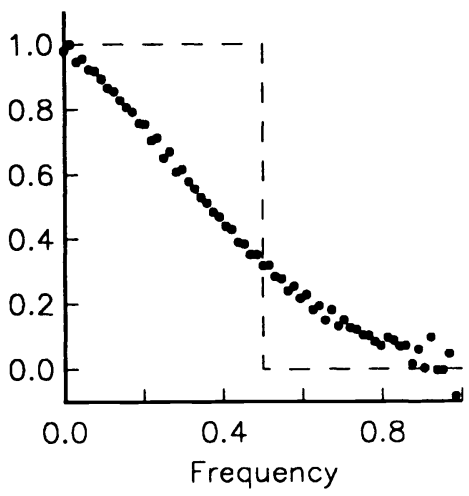

(a)

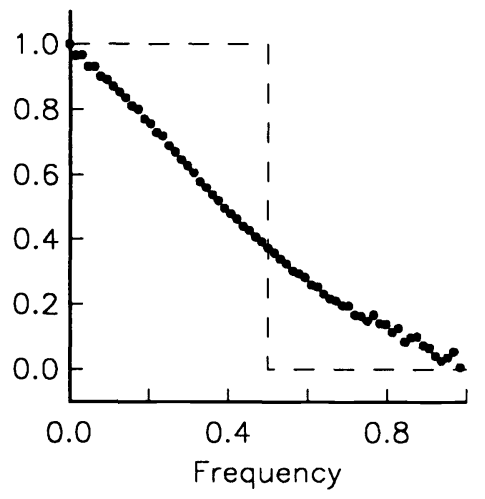

(b)

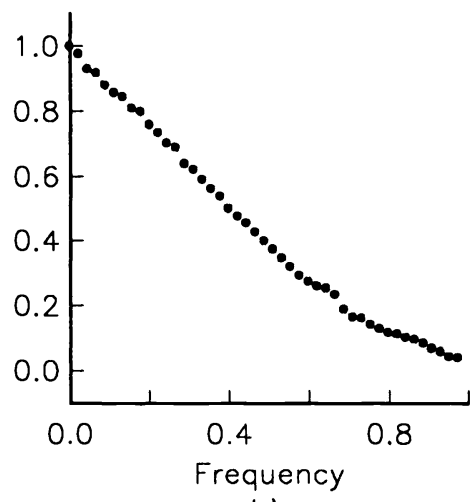

(c)

Fig. 8. Estimates of CCD system OTF. (a) Vertical estimate. (b) Horizontal estimate. (c) Diagonal estimate.

frequencies on the bar chart ranged from 1 cycle/mm to 5.6 cycles $/ \mathrm{mm}$. The sampling frequency in the target plane was 6 samples $/ \mathrm{mm}$, so the normalized square-wave frequencies relative to the sampling frequency ranged from 0.167 to 0.933 . From the OTF estimate of Fig. 8(b), the modulation or contrast (i.e., the standard deviation) in the images of the square waves is predicted by the equation ${ }^{24}$

$\hat{g}(u)=\frac{2 a}{\pi}\left[\sum_{k=0}^{\infty}\left|\frac{\hat{h}(u(2 k+1))}{2 k+1}\right|^{2}\right]^{1 / 2}$,

where $\hat{g}$ is the estimated contrast for a square wave of frequency $u$ and $a$ is a scaling constant. Figure 9 shows the normalized predicted contrast (solid line) and the observed contrast (closed circles) of the square-wave images. The OTF estimate accurately predicts the observed square-wave contrast. The scatter of the observed contrast is due to noise and sample/scene phase. Only three bars were usable to all frequencies, and with so few periods of the square wave, sample/scene phase is a source of significant variability in the observed contrast.

\section{CONCLUSION}

The extended knife-edge technique can accurately estimate the OTF of a digital imaging system. By registering many scans to create a superresolution scan, the extended technique can estimate the OTF beyond the Nyquist limit of the device. Scan-line averaging successfully suppresses noise and increases SNR, making the estimates more accurate and less variable. The technique can be applied at virtually any angle to generate twodimensional OTF estimates or assess the symmetry and separability of the OTF.

\section{ACKNOWLEDGMENTS}

This research was supported by a NASA Graduate Research Fellowship and a National Research Council-NASA Research Associateship at Langley Research Center in Hampton, Va., and by the Center for Communication and Information Science at the University of Nebraska - Lincoln. The authors are grateful for the support and the help of Friedrich O. Huck and Judith A. McCormick.

\section{REFERENCES}

1. R. V. Shack, "Characteristics of an image-forming system," J. Res. Nat. Bur. Stand. 56(5), 245-260 (1956).

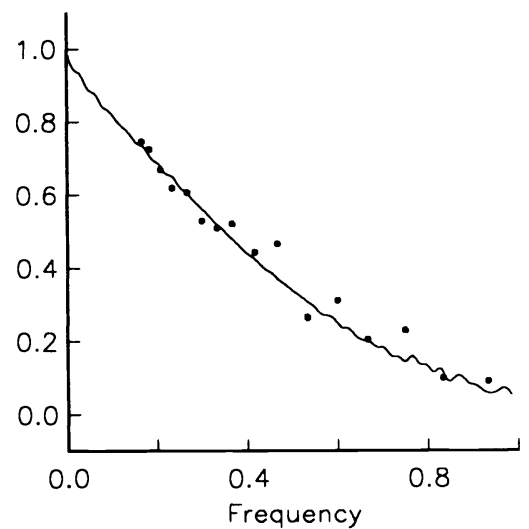

Fig. 9. Square-wave estimated and observed contrast.

2. G. C. Higgins, "Methods for engineering photographic systems," Appl. Opt. 3(1), 1-10 (1964)

3. R. Barakat and A. Houston, "Line spread function and cumulative line spread function for systems with rotational symmetry," JOSA 54(6), 768-773 (1964).

4. B. Tatian, "Method of obtaining the transfer function from the edge response function," JOSA 55(8), 1014-1019 (1965).

5. R. Barakat, "Determination of the optical transfer function directly from the edge spread function," JOSA 55(10), 1217-1221 (1965).

6. R. A. Jones, "An automated technique for deriving MTFs from edge traces," Photog. Sci. Eng. 11(2), 102-106 (1987)

7. R. A. Jones and E. C. Yeadon, "Determination of the spread function from noisy edge scans. Photog. Sci. Eng. 13(4), 200-204 (1969).

8. M. E. Rabedeau, "Effect of truncation of line-spread and edge-response functions on the computed optical transfer function," JOSA 59(10), 1309-1314 (1969).

9. E. S. Blackman, "Recovery of system transfer functions from noisy photographic records," in Image Information Recovery, Proc. SPIE 16, 105-112 (1969).

10. E. C. Yeadon, R. A. Jones, and J. T. Kelly, "Confidence limits for individual modulation transfer function measurements based upon the phase transfer function," Photog. Sci. Eng. 14(2), 153-156 (1970).

11. A. G. Tescher and H. C. Andrews, "Data compression and enhancement of sampled images," Appl. Opt. 11(4), 919-925 (1972).

12. P. L. Smith, "New technique for estimating the MTF of an imaging system for its edge response," Appl. Opt. 11(6), 1424-1425 (1972).

13. R. M. Simonds, "Two dimensional modulation transfer functions of image scanning systems," Appl. Opt. 20(4), 619-622 (1981).

14. W. F. Schreiber, Fundamentals of Electronic Imaging Systems: Some Aspects of Image Processing, Srpinger-Verlag, New York (1986).

15. S. K. Park and R. A. Schowengerdt, "Imaging sampling, reconstruction, and the effect of sample-scene phasing," Appl. Opt. 21(17), 3142-3151 (1982).

16. S. K. Park, R. A. Schowengerdt, and M. A. Kaczynski, "Modulation transfer function analysis for sampled image systems," Appl. Opt. 23(15), 2572-2582 (1984).

17. G. Béal, G. Boucharlat, J. Chabbal, J. P. Dupin, B. Fort, and Y. Mellier, "Thomson-CSF frame-transfer charge-coupled-device imagers: design and evaluation at low flux level,'’ Opt. Eng. 26(9), 902-910 (1987). 
18. A. J. Tabatabai and O. R. Mitchell, "Edge location to subpixel values in digital imagery,' IEEE Trans. Patt. Anal. Machine Intell. 6(2), 188-201 (1984).

19. E. M. Mikhail, M. L. Akey, and O. R. Mitchell, "Detection and subpixel location of photogrammetric targets in digital images," Photogrammetria 39(3), 63-83 (1984).

20. M. Mazumdar, B. K. Sinha, and C. C. Li, "Comparison of several estimates of edge point in noisy digital data across a step edge," in Proc. Conf. on Computer Vision and Pattern Recognition, pp. 27-33, IEEE Computer Society (1985).

21. P. Seitz, "Optical superresolution using solid-state cameras and digital signal processing," Opt. Eng. 27(7), 535-540 (1988).

22. Y. Talmi and R. W. Simpson, "Self-scanned photodiode array: a multichannel spectrometric detector,' Appl. Opt. 19(9), 1401-1414 (1980).

23. R. K. Hopwood, "Design considerations for a solid-state image sensing system," in Minicomputers and Microprocessors in Optical Systems, Proc. SPIE 230, 72-82 (1980).

24. R. W. Hamming, Digital Filters, Second Edition, Prentice-Hall, Englewood Cliffs, N.J. (1983).

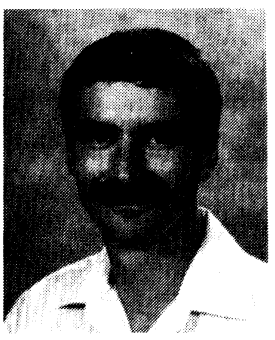

Stephen E. Reichenbach earned a bachelor's degree in English from the University of $\mathrm{Ne}$ braska-Lincoln (1976) and a master's degree in computer science from Washington University in Saint Louis (1984). From 1986 to 1989 , he attended the College of William and Mary, where he was a graduate assistant at the Virginia Institute of Marine Science (1986-87) and a recipient of a NASA Graduate Researcher Fellowship (1987-89). In 1989, he completed his dissertation, "Small-kernel image restoration" and earned a doctoral degree in computer science. After receiving his doctorate, he was awarded a National Research Council Research Associateship in the Visual Information Processing Laboratory at the NASA Langley Research Center (1989-90). $\mathrm{He}$ is currently an assistant professor in the Computer Science and Engineering Department at the University of Nebraska - Lincoln (email: reich@fergvax.unl.edu), where his research interests are digital image restoration and reconstruction, image coding and compression, and automated image analysis.

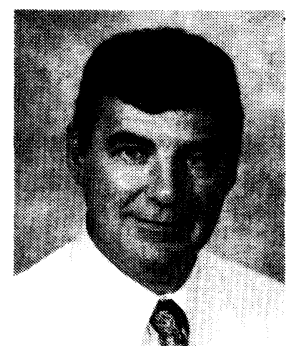

Stephen K. Park has been a professor of computer science at the College of William and Mary since 1986. Prior to this, he was a member of the research staff at NASA Langley Research Center. He received a doctorate in applied mathematics from North Carolina State University in 1970. Dr. Park is a member of the Association of Computing Machinery and the IEEE Computer Society. His research interests include the modeling, simulation, and performance analysis of digital imaging systems; algorithms for the reconstruction and restoration of sampled data; the conceptual design and simulation of computer vision systems; and stochastic methods in discrete event systems simulation. He has published more than 50 research papers in these and related areas. His email address is park@cs.wm.edu.

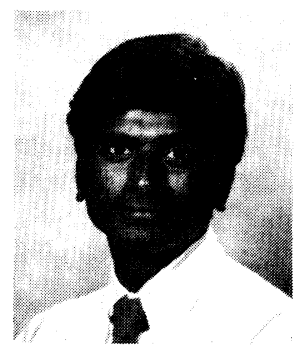

Ramkumar Narayanswamy received his BS degree in electrical engineering from the University of Madras, India, in 1984 and his MS degree from the University of Virginia in 1986. $\mathrm{He}$ has since been working as a research engineer for Science and Technology Corporation. He is currently based at NASA Langley Research Center and is working in the area of visual information processing. His interests include image restoration, image coding, and feature extraction. 